Case Report

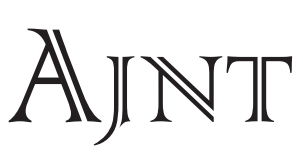

\title{
Isolated Renal Sarcoidosis: a Rare Cause for Granulomatous Interstitial Nephritis
}

\author{
Amal Abdel Ghani ${ }^{a^{*}}$, Salah Al Waheeb ${ }^{\mathrm{b}}$ and Ekhlas Al Homoud ${ }^{\mathrm{c}}$ \\ a. Nephrology Unit, b. Department of Histopathology, c. Department of Medicine, Mubarak Al Kabeer Hospital, Kuwait
}

\begin{abstract}
Introduction: Granulomatous interstitial nephritis (GIN) is a rare histological diagnosis that can be the first manifestation of systemic disease. It has been associated with medications, infections, sarcoidosis, crystal deposits, paraproteinemia and Wegnere's granulomatosis. It can also be seen in an idiopathic form.
\end{abstract}

Case report: A 37 year-old female patient presented to our nephrology service with lethargy and weight loss of three months duration. Her past medical history and clinical examination were not remarkable. She had impaired renal function tests and hypercalcemia associated with elevated angiotensin converting enzyme levels and sonographically normal kidneys. Extensive investigations revealed no other organ involvement and no evidence of infection or malignancy. Renal biopsy showed extensive interstitial non-necrotizing granulomata. Accordingly, she was diagnosed as a case of isolated renal sarcoidosis and started on steroid therapy. Within one month of treatment, she recovered normal renal function. Steroids were successfully tapered after another six months, and she shows no evidence of recurrence 24 months after steroid withdrawal.

Conclusion: The possibility of isolated renal sarcoidosis should be considered in any case of impaired kidney function and hypercalcemia even in the absence of systemic manifestation. The finding of non-caseating interstitial granulomata in renal biopsy supports this diagnosis.

* Corresponding author; Mubarak Al kabeer Hospital, Nephrology department, P.O Box 43787, Code 3205, Hawally, Kuwait

E mail: Amalhassan90@hotmail.com
Keywords: granulomatous interstitial nephritis, non-caseating granuloma, renal insufficiency, renal sarcoidosis.

\section{Introduction}

Granulomatous interstitial nephritis (GIN) is a rare histological diagnosis that is present in between $0.5-0.9 \%$ of native renal biopsies and $0.6 \%$ of renal transplant biopsies [1-3]. GIN has been associated with medications, infections, sarcoidosis, crystal deposits, paraproteinemia and Wegnere's granulomatosis but can also be seen in an idiopathic form [4]. GIN may be the first manifestation of systemic diseases such as sarcoidosis [5].

\section{Case history}

A 37 year-old female patient presented to our nephrology service with lethargy and weight loss of three months duration. She also complained of hair loss and joint pains for which she was using non-steroidal anti-inflammatory drugs. She had no significant past medical history and clinical examination was not remarkable with no evidence of arthritis or joint deformities, and no mucocutaneous manifestations.

Routine blood investigations were normal apart from renal function tests; which showed BUN of $43.97 \mathrm{mg} /$ $\mathrm{dl}$, serum creatinine $5.43 \mathrm{mg} / \mathrm{dl}$, serum bicarbonate 17.8 $\mathrm{mmol} / \mathrm{l}$, corrected serum calcium $12.68 \mathrm{mg} / \mathrm{dl}$, serum phosphorus $5.26 \mathrm{mg} / \mathrm{dl}$, and serum alkaline phosphatase $68 \mathrm{IU} / 1$. Urine analysis was not informative; microscopy showed no active sediment and no detectable acid-fast bacilli, urine culture including special culture technique for acid fast bacilli was negative, and testing for Bence Jones protein was negative. She had sonographically normal kidneys, negative skeletal survey, normal high resolution CT scan of the chest and abdomen, negative PPD test, and normal serum protein electrophoresis pattern. Blood tests for tumor markers, immunology screening 
Figure 1: Histopathological sections of the kidney showing non-caseating granulomata

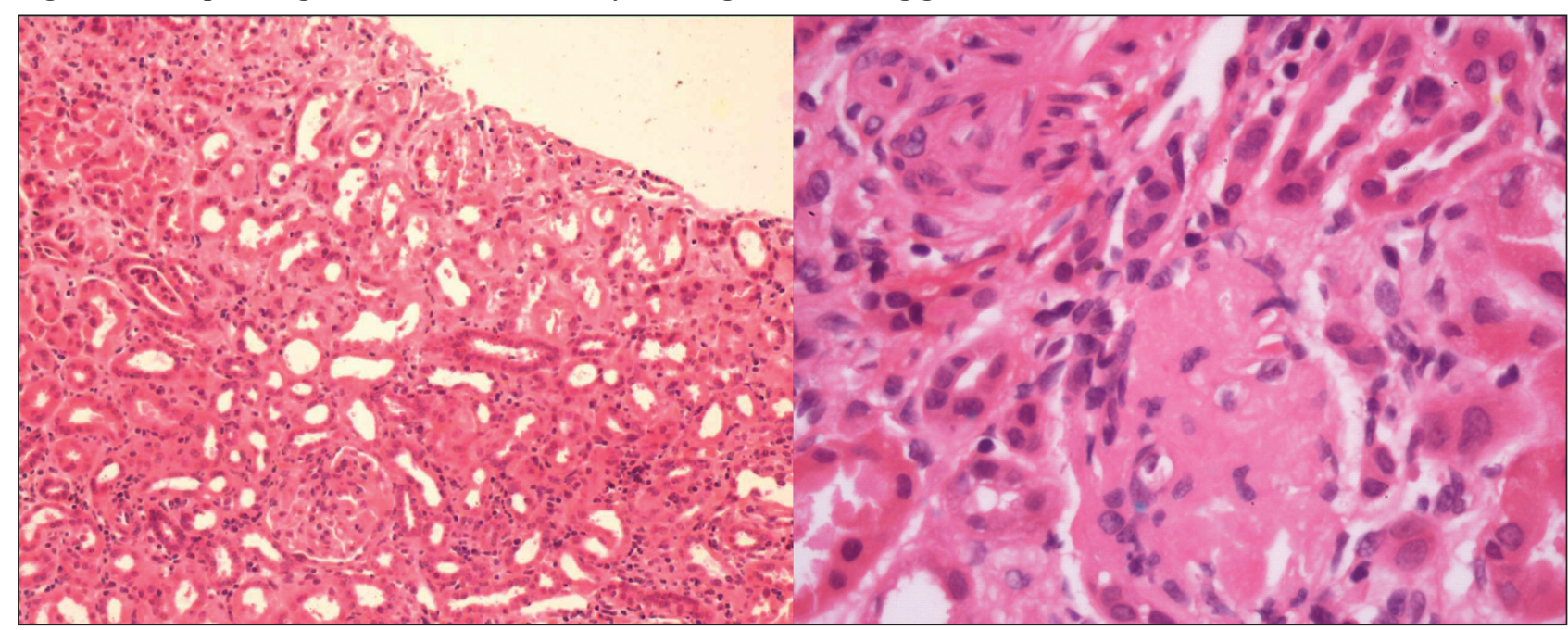

and culture and sensitivity were all negative. However, she had elevated serum angiotensin converting enzyme levels. Renal biopsy showed normal glomeruli with extensive tubulointerstitial infiltration by lymphocytes and multinucleated giant cells, forming interstitial nonnecrotizing granulomata. Immune staining was negative as well as staining for acid fast bacilli (Figure 1).

The possibility of sarcoid granuloma was raised and the patient was started on oral prednisolone $40 \mathrm{mg} / \mathrm{d}$. Renal function started to improve within one week of steroid treatment, and after one month her results showed BUN $12.88 \mathrm{mg} / \mathrm{dl}$, serum creatinine $0.96 \mathrm{mg} / \mathrm{dl}$ and serum corrected calcium $9.2 \mathrm{mg} / \mathrm{dl}$. Treatment was tapered slowly after another six months and the patient maintained on regular follow up. After about 24 months of steroid withdrawal, she still has normal renal function tests and normal serum calcium level.

\section{Discussion}

Sarcoidosis is a multi system disease in which the kidney may be involved [3]. It is characterized by the formation of non-caseating granulomata in various organs. The hallmark of the disease, sarcoid granuloma, was first described by Schaumann [6]. The sarcoid granuloma is a rounded cellular collection of epitheloid cells and multinucleated giant cells surrounded by a rim of lymphocytes and macrophages in association with various degrees of fibrosis but without caseation [4].

The first case of isolated renal sarcoidosis was described in 1976 by Spargo [7]. Since that time, only 100 cases of isolated renal sarcoidosis have been described in the literatures [8]. Sarcoidosis can affect the kidney through various mechanisms. These include hypercalcemia caused by overproduction of vitamin D by lymphocytes, granulomatous infiltration of the interstitium, a variety of glomerulopathies, and other miscellaneous conditions that include type AA amyloidosis and renal artery stenosis (sarcoid angitis) [9]. Hypercalcemia can affect the kidney through its hemodynamic effect which may result in reduction of the glomerular filtration rate, impairment of urinary concentration ability, nephrocalcinosis $(<5 \%)$ or nephrolithiasis $(<10 \%)$ leading to obstructive uropathy [9].

Steroid therapy is the mainstay of treatment. Beside a possible direct effect on the disease process, steroids block the extra renal calcitriol production and ameliorate the associated hypercalcemia. Steroids should be given for six months to one year and usually produce an excellent response [10].

Other treatment options include aminoquinolone derivatives, calcium binding agents, cyclophosphamide, thalidomide, pentoxyphylline, infleximab, cyclosporine, methotrexate, and azathioprine $[11,12]$.

\section{Conclusion}

The possibility of isolated renal sarcoidosis should be considered in any case of impaired kidney function associated with unexplained hypercalcemia even in the absence of extra-renal systemic manifestations. The finding of granulomatous interstitial nephritis in renal biopsy supports this diagnosis. 


\section{References}

1. O'Riorden E, Willert RP, Reeve R, Kalra PA, O'Donoghue DJ, Foley RN, Waldek. Isolated sarcoid granulomatous interstitial nephritis: Review of five cases at one center. Clin Nephrol. 2001 Apr; 55(4):297-302.

2. Joss N, Morris S, Young B, Geddes C. Granulomatous interstitial nephritis. Clin J Am Soc Nephrol. 2007 Mar;2(2):222-30.

3. Meehan SM, Josephson MA, Haas M. granulomatous tubulointerstitial nephritis in the renal allograft. Am J Kidney Dis. 2000 Oct;36(4):E27.

4. Agostini C, Adami F, Semenzato G. New pathogenetic insights into the sarcoid granuloma. Curr Opinion Rheumatol. 2000 Jan;12(1):71-6.

5. Nunes H, Bouvry D, Soler P, Valeyre D. Sarcoidosis. Orphanet J Rare Dis. 2007 Nov 19;2:49.

6. Schaumann J. Lymphogranuloma benigna in the light of prolonged clinical observations and autopsy findings. Br J Dermatol. 1936;48:399-46.
7. Javaud N, Belenfant X, Stirnemann J,Laederich J, Ziol M, Callard P, Ronco P, Rondeau E, Fain O. Renal granulomatoses, a retrospective study of 40 cases and review of the literature. Medicine (Baltimore). 2007 May;86(3):170-80.

8. Miyoshi K, Okura T, Manabe S, Watanabe S, Fukuoka T, Higaki J. Granulomatous interstitial nephritis due to isolated renal sarcoidosis. Clin Exp Nephrol. 2004 Sep;8(3):279-82.

9. Robsom MG, Banerjee D, Hopster D, Cairns HS. Seven cases of granulomatous interstitial nephritis in the absence of extrarenal sarcoid. Nephrol Dial Transplant. 2003 Feb;18(2):280-4.

10. Grutters JC, van den Bosch JM. Corticosteroid treatment in sarcoidosis. Eur Respir J. 2006 Sep;28(3):627-36.

11. BaughmanRP.Therapeuticoptions forsarciodosis:new and old. Curr Opin Pulm Med. 2002 Sep;8(5):464-9.

12. Thumfart J, Muller D, Rudolph B, Zimmering M, Querfeld U, Haffner D. Isolated sarcoid granulomatous interstitial nephritis responding to infleximab therapy. Am J Kidney Dis. 2005 Feb;45(2):411-4. 